\title{
Surface energy and work function of the light actinides
}

\author{
Kollár, J.; Vitos, Levente; Skriver, Hans Lomholt
}

Published in:

Physical Review B

Link to article, DOI:

10.1103/PhysRevB.49.11288

Publication date:

1994

Document Version

Publisher's PDF, also known as Version of record

Link back to DTU Orbit

Citation (APA):

Kollár, J., Vitos, L., \& Skriver, H. L. (1994). Surface energy and work function of the light actinides. Physical Review B, 49(16), 11288-11292. https://doi.org/10.1103/PhysRevB.49.11288

\section{General rights}

Copyright and moral rights for the publications made accessible in the public portal are retained by the authors and/or other copyright owners and it is a condition of accessing publications that users recognise and abide by the legal requirements associated with these rights.

- Users may download and print one copy of any publication from the public portal for the purpose of private study or research.

- You may not further distribute the material or use it for any profit-making activity or commercial gain

- You may freely distribute the URL identifying the publication in the public portal

If you believe that this document breaches copyright please contact us providing details, and we will remove access to the work immediately and investigate your claim. 


\title{
Surface energy and work function of the light actinides
}

\author{
J. Kollár and L. Vitos \\ Research Institute for Solid State Physics, P.O. Box 49, H-1525 Budapest, Hungary \\ H. L. Skriver \\ Center for Atomic-Scale Materials Physics and Physics Department, Technical University of Denmark, \\ DK-2800 Lyngby, Denmark
}

(Received 21 December 1993)

\begin{abstract}
We have calculated the surface energy and work function of the light actinides Fr, Ra, Ac, Th, $\mathrm{Pa}, \mathrm{U}, \mathrm{Np}$, and $\mathrm{Pu}$ by means of a Green's-function technique based on the linear-muffin-tin-orbitals method within the tight-binding representation. In these calculations we apply an energy functional which combines the kinetic energy calculated within the atomic-sphere approximation with Coulomb- and exchange-correlation-energy terms calculated by means of the complete nonspherically symmetric charge density derived from the atomic-sphere potential within nonoverlapping and space-filling cells. The calculated surface energies and work functions are in good agreement with the limited experimental data.
\end{abstract}

\section{INTRODUCTION}

The surface energy and the work function are the two most fundamental electronic properties of a metallic surface, and their determination is of great importance in the understanding of a wide range of surface phenomena. For most metals the work function and the surface energy are known from experiments, although the recommended values appear to have uncertainties of unknown magnitude. This, for instance, is true for the surface energies derived from the surface tension of liquid metals and listed by de Boer et al. ${ }^{1}$ Nonetheless, to our knowledge the compilation by these authors represents the most consistent choice of surface energies in existence. Similarly, the work functions listed by Michaelson ${ }^{2}$ are derived mainly from measurements on polycrystalline samples and in many cases have not been confirmed by ultrahigh vacuum measurements. For the actinides the situation is even less satisfactory in that the work functions and surface energies are only known for three out of the first eight metals in the series. In this situation one may turn to theoretical models and in particular to $a b$ initio calculations as a guide, especially since the accuracy of such calculations has recently improved considerably.

Here, we present calculations of the surface energy and the work function of the fcc (111) surface of the light actinides including also $\mathrm{Fr}$ and $\mathrm{Ra}$. For this purpose, we first applied the tight-binding linear muffin-tin orbitals (LMTO) Green's-function technique ${ }^{3}$ within the atomic sphere approximation (ASA), which was successfully used in the calculation of surface properties of a large series of metals. ${ }^{4,5}$ Since the early actinides have an $f$-band, which is either just above the Fermi level (Ac, Th) or occupied (Pa-Pu), we included $s, p, d$, and $f$ orbitals in the calculation resulting in slightly negative surface energies for Ac and Th. This unphysical outcome for the early actinides led us to develop a more accurate functional based on the ASA for the kinetic energy and on the complete charge density, derived from the spherically symmetric atomic sphere potential, for the Coulomb and exchange-correlation energies. ${ }^{6}$ The functional has been tested in calculations of the surface energy of the $4 d$ transition metal series ${ }^{6}$ and now the mean deviation from the full-potential calculations ${ }^{7}$ is less than $4 \%$. Here we present surface energies and work functions for the early actinides calculated by means of this improved functional and expect the results to be of the same accuracy as a full-potential calculation.

\section{COMPUTATIONAL METHOD}

The LMTO Green's-function technique as implemented by Skriver and Rosengaard ${ }^{3}$ is based on the work of Andersen and co-workers. ${ }^{8-14}$ An essential aspect of the technique is the ability, within the ASA and in the tight-binding representation, to generate the Green'sfunction matrices for a real, two-dimensional interface by a simple and efficient procedure. In addition, the ASA allows an efficient and accurate determination of the kinetic energy and also of the Coulomb and exchange-correlation energies if, as was shown in Ref. 6, one goes beyond the ASA for the charge-density and multipole moments.

In the present implementation we combine the ASA kinetic energy $T_{\text {ASA }}$ with accurately treated Coulomb and exchange-correlation energy terms evaluated by means of the complete nonspherically symmetric charge density derived from the ASA potential within nonoverlapping, space-filling cells. ${ }^{6}$ The full-charge-density (FCD) energy functional may be written

$$
E_{\mathrm{FCD}}[n(\mathbf{r})]=T_{\mathrm{ASA}}+F[n(\mathbf{r})],
$$

where $n(\mathbf{r})$ is the electronic charge density and the functional $F$ may be partitioned into cell-contributions 
$F=\sum_{\mathbf{R}} F^{\mathbf{R}}$ each of which is the sum of intercell (Madelung), intracell Coulomb, and exchange-correlation energy terms

$F^{\mathbf{R}}=\sum_{\mathbf{R}^{\prime} \neq \mathbf{R}} E_{\text {inter }}^{\mathbf{R}}\left[Q_{L}^{\mathbf{R}}, Q_{L}^{\mathbf{R}^{\prime}}\right]+E_{\text {intra }}^{\mathbf{R}}\left[n^{\mathbf{R}}\right]+E_{\mathbf{x c}}^{\mathbf{R}}\left[n^{\mathbf{R}}\right]$.

Here, ${ }^{\mathbf{R}}$ is the electronic charge density inside the nonoverlapping, space-filling cell centered at $\mathbf{R}$ obtained from a self-consistent ASA potential. The multipole moments of the cells are defined as

$$
Q_{L}^{\mathbf{R}}=\frac{(4 \pi)^{1 / 2}}{2 l+1} \int_{\text {WS cell }}\left(\frac{r}{S}\right)^{l} n^{\mathbf{R}}(\mathbf{r}) Y_{L}(\hat{\mathbf{r}}) d \mathbf{r}
$$

in terms of the atomic Wigner-Seitz radius $S$ and the real harmonics $Y_{L}$. A detailed description of the calculation of the intercell, intracell, and exchange-correlation contributions is given in Ref. 6 .

The surface energy is the work required to form a unit area of surface, and is the solid analog of the surface tension of a liquid. In the present case and within the BornOppenheimer, local-density, and frozen-core approximations it is simply calculated as the difference

$$
\gamma=E_{\mathrm{FCD}}^{2 \mathrm{D}}(N)-N E_{\mathrm{FCD}}^{3 \mathrm{D}}
$$

between the total energy of the $N$ atoms in the surface region (2D) and $N$ times the total energy of a single atom in the bulk (3D).

Within the ASA, and for a bulk cell with only equivalent atoms, the zero of energy is taken to coincide with the electrostatic potential at the surface of the (neutral) atomic sphere, and the work function $W$ of the corresponding semi-infinite metal may then be expressed as the difference

$$
W=\phi-E_{F}
$$

between the electrostatic surface dipole $\phi$ and the Fermi level $E_{F}$ for the bulk crystal on the ASA energy-scale.

At the outset of a surface calculation, one needs starting potentials as well as total energies corresponding to the perfect, infinite crystal of the atomic species that form the surface. To obtain this input, we perform selfconsistent bulk calculations by means of the second order LMTO Hamiltonian also used in the Green's-function technique, and calculate the kinetic energy by integrating the Green's function on a complex energy contour. Based on convergence tests we use a surface region consisting of four layers of metal plus two layers of empty spheres simulating the vacuum. For the $k$-space integration we use 45 special points ${ }^{15}$ in the irreducible part of the two-dimensional Brillouin zone for the fcc (111) surface. Finally, we calculate the moments of the state density by means of a Gaussian integration technique with 16 points distributed exponentially on a semicircle in the complex plane to emphasize the contributions close to the Fermi level. For the exchange-correlation potential and energy we use the Perdew-Zunger interpolation ${ }^{16}$ of the many-body calculations of Ceperley and Alder. ${ }^{17}$

\section{RESULTS}

The electronic structure of the light actinides is formed by $s p d$ bands, as in an ordinary $d$ transition metal, plus an $f$ band which descends and becomes occupied in Pa. This is illustrated in Fig. 1, where we show the position and the width of the bands obtained in the scalarrelativistic, bulk LMTO-ASA calculations. In addition to showing the descend of the $5 f$ band, the figure illustrates that beyond $\mathrm{Ra}$ the $6 p$ states cannot be treated as core states and must be included in the band calculation. Thus, in our bulk and surface calculations the band structure is obtained in two panels, one including the $6 p$ (semicore) and the other the $7 p$ states. Here, we neglect the sizeable spin-orbit splitting of the $6 p$ states but for a completely filled band this will have little effect on the surface properties.

The effect of the $6 p$ semicore on the surface energy may be estimated by arguments of the kind used in the initialstate theory of surface core level shifts in $d$ transition metals. ${ }^{18}$ In the present case these arguments imply that the center of the $6 p$ semicore states may approximately be identified with the bulk-to-surface shift of the valence $5 f$ band. At the surface the $5 f$ band becomes narrow owing to the decrease in coordination number, and if one assumes an approximate conservation of the $f$ charge in each layer, to avoid the large electrostatic energy, the surface $f$ band must be positioned below the bulk $f$ band for an $f$ occupation $n_{f}<7$ and above the bulk $f$ band for $n_{f}>7$. As a result, we expect that at the beginning of the light actinide series the $6 p$ band in the surface layer will be shifted downwards relative to its bulk position and that this shift will decrease as the $5 f$ filling increases.

In Fig. 2 we have plotted the bulk to surface shift of the center of gravity of the $6 p$ band for the light actinides. The result is completely in accordance with the picture discussed above and leads to a negative $6 p$ semicore contribution to the sum of the one-electron energies and hence to the surface energy of these metals. In Th the $6 p$ semicore contribution is $-0.3 \mathrm{~J} / \mathrm{m}^{2}$ and causes a

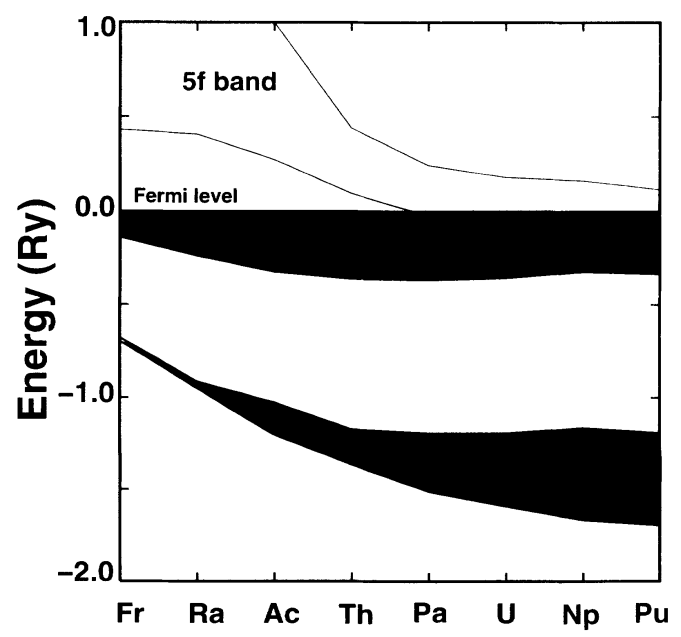

FIG. 1. The energy band structure of the light actinides. The band positions are measured from the actual Fermi level. 


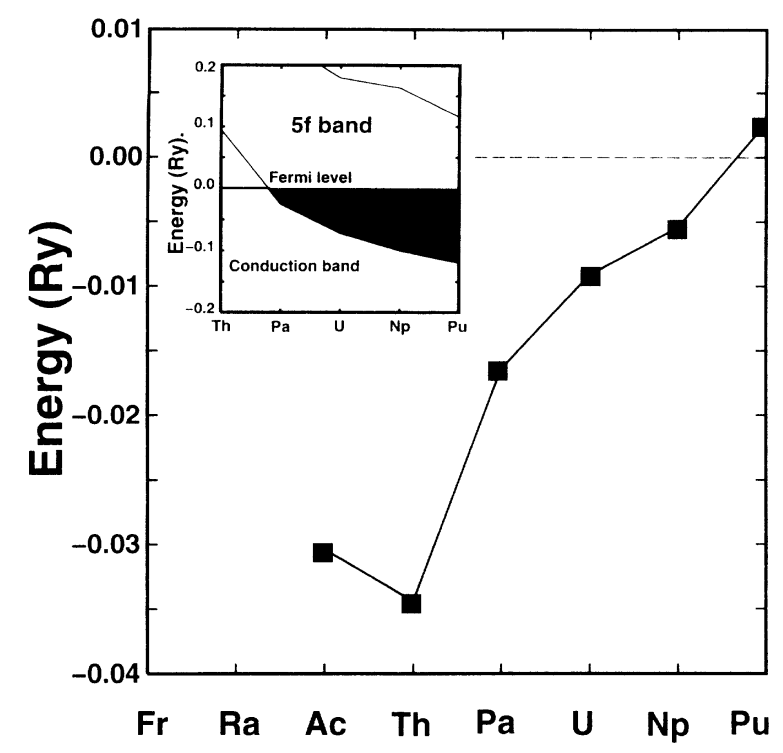

FIG. 2. The surface shift of the center of gravity $C_{6 p}$ of the completely occupied $6 p$ band. The inset shows the filling of the $5 f$ conduction band.

$30 \%$ reduction in the calculated surface energy. The effect is smaller in the other actinides and disappears in $\mathrm{Pu}$.

The results of calculations by the Green's-function technique in the original implementation, ${ }^{3,5}$ which is based on a spherically symmetric potential and charge density but in addition includes the dipole contribution to the (Madelung) intersphere potential, are shown in Fig. 3. These results include the contribution from the

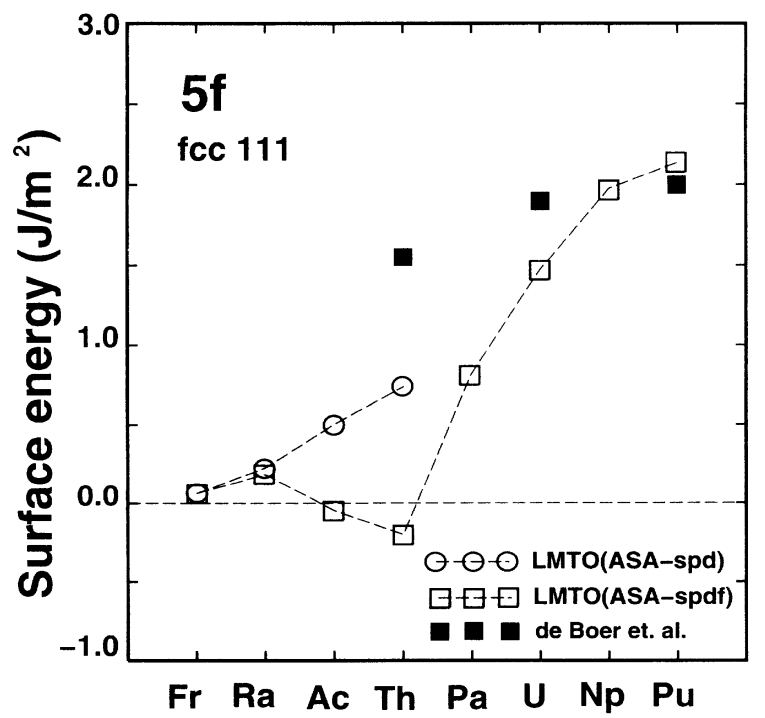

FIG. 3. The surface energy of the light actinides calculated within the ASA but including the dipole contribution to the intersphere potential. For Fr-Th the results obtained by means of an $s, p, d$ basis are also shown together with the semiempirical values by de Boer et al. (Ref. 1).

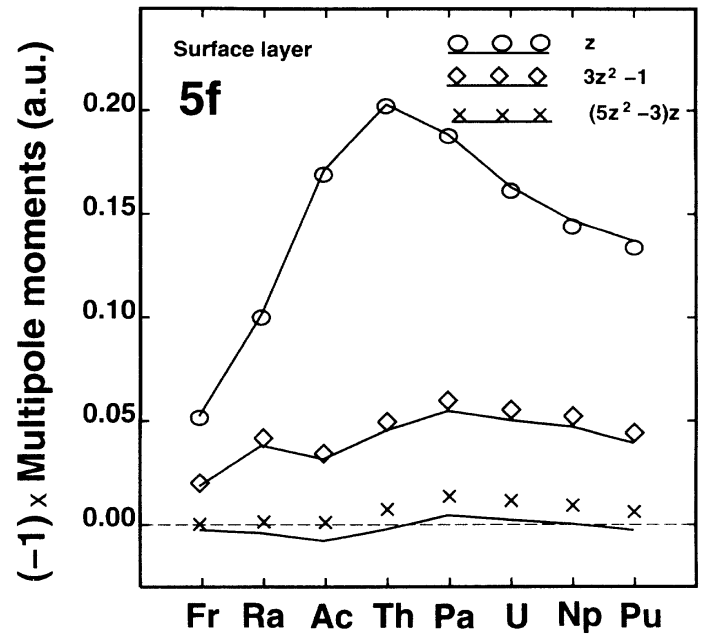

FIG. 4. The multipole moments $Q_{L}$ with $L=p, d, f$ in the surface layer of the fcc (111) face of Fr through $\mathrm{Pu}$.

$6 p$ semicore and for lack of a better word will be referred to as an ASA calculation. As may be seen, the ASA gives too low surface energies at the beginning of the series. For Ac and Th the calculated surface energy is even negative and, hence, unphysical. The reason for this failure is mainly the fact that by the introdution of $f$-states the calculational technique has more degrees of freedom and produces a larger dipole moment at the surface, even in the case of Th which is essentially a $d$ band metal. The neglect of the Coulomb interaction of the higher multipole moments therefore leads to larger errors than is usually seen in spd ASA calculations. The

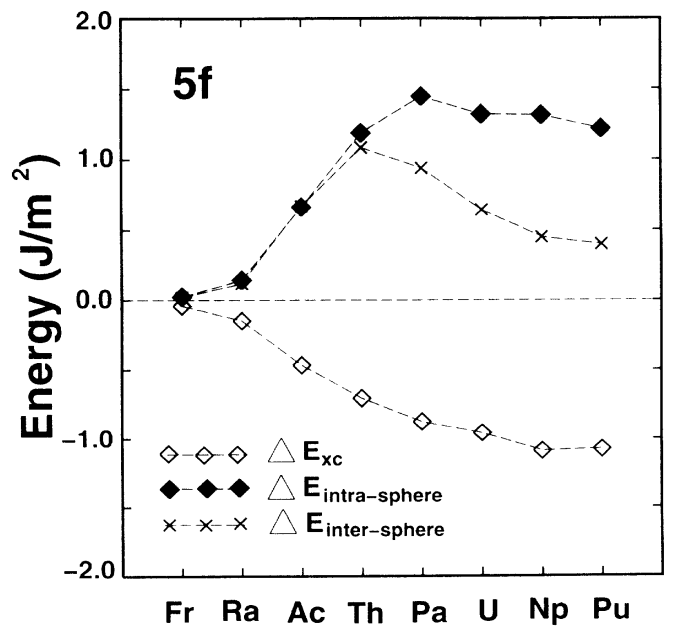

FIG. 5. The nonspherical contributions to the surface energy plotted as the difference between the full charge-density results and ASA results for the intrasphere and intersphere Coulomb terms as well as the exchange-correlation energy. Note that in this context the ASA results include also the monopole-dipole intersphere (Madelung) contribution. 


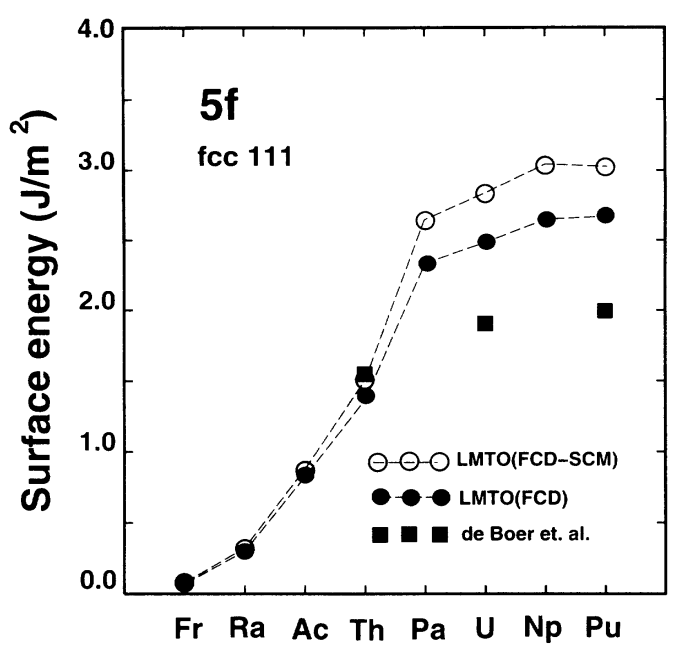

FIG. 6. The surface energy of the light actinides for the fcc (111) face calculated within our FCD scheme using spherical cells (SCM) and nonoverlapping, space-filling WS cells. The values are compared to the semiempirical results (Ref. 1 ).

$l$-convergence of the LMTO-Green's-function technique and the solution to this problem is discussed in detail in Ref. 6.

In Fig. 4 we present the $p, d$, and $f$ multipole moments calculated in the spherical cell model (SCM) defined in Ref. 6 as well as by integration within nonoverlapping and space-filling cells. ${ }^{6}$ In the SCM, as in the ASA, we assume that the lattice consists of atomic spheres, the overlap of which is neglected, but now the charge density is not necessarily spherically symmetric within the sphere. It may be seen in the figure that the SCM already gives fairly good values for the higher multipole moments, and that the dipole moment which attains a maximum value at Th plays a dominant role. It is this high value of the dipole moment and the neglect in the original Green'sfunction technique of the dipole-dipole interactions which is mainly responsible for the low calculated surface energy in Ac and Th.

In Fig. 5 we show the nonspherical intercell, intracell, and exchange-correlation energy contributions defined in (2) to the surface energy, i.e., their values obtained in the FCD calculation relative to those of the ASA. It is seen that the increase in the electrostatic contributions is not completely compensated by a corresponding decrease in the exchange-correlation energy, which results in an overall positive correction which has its maximum value at $\mathrm{Th}$.

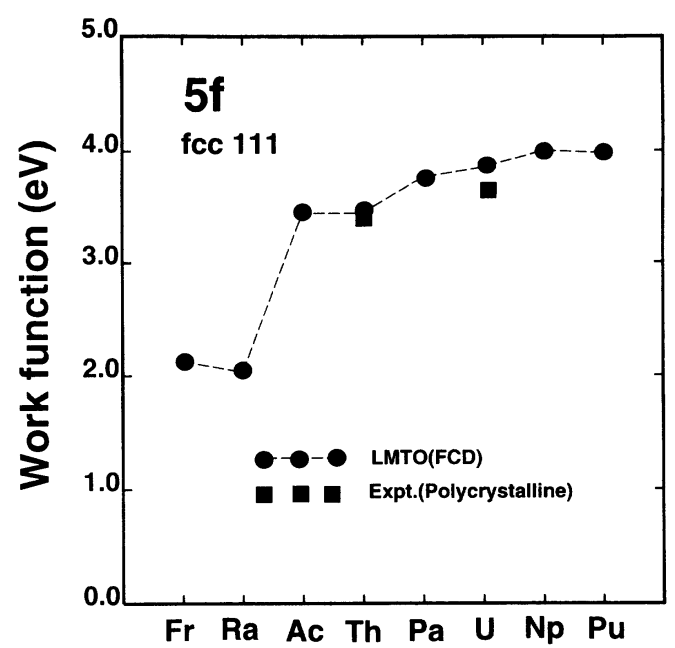

FIG. 7. Work function calculated in our FCD scheme compared to the available experimental data (Ref. 2).

The result of the FCD calculation of the surface energy of the light actinides is plotted in Fig. 6 together with the available semiempirical values by de Boer et al. ${ }^{1}$ It may be seen that the deficiencies of the original Green'sfunction technique have disappeared and that the calculation reproduces the experimental trend and to within $20 \%$ reproduces the semi-empirical values for $\mathrm{U}$ and $\mathrm{Pu}$. The figure also includes the results of the SCM calculation which generally are larger than the FCD results owing to the overestimate of the higher multipoles seen in Fig. 4.

In Fig. 7 we present the calculated work functions of the light actinides and compare them to the measured values available for thorium and uranium. In both cases the agreement is excellent. Recently, Hao and coworkers ${ }^{19,20}$ calculated the surface electronic structure of $\gamma-\mathrm{U}$ and $\delta$-Pu. They estimated the work function of $\mathrm{U}$ to be in the range from 3.60 to $3.82 \mathrm{eV}$ and for $\mathrm{Pu}$ they found 3.68 and $4.14 \mathrm{eV}$ for the (100) and (111) faces, respectively. Again these values are in good agreement with our results. The FCD calculations of surface energy and work function are summarized in Table I.

\section{ACKNOWLEDGMENTS}

This work was supported in part by Grant No. ERBCIPA-CT-92-2096 of the Commission of the European

TABLE I. Calculated atomic Wigner-Seitz radii $S_{\mathrm{Ws}}$, surface energy $\gamma$, and work function $W$ for the light actinides.

\begin{tabular}{lcccccccc}
\hline \hline & Fr & $\mathrm{Ra}$ & $\mathrm{Ac}$ & $\mathrm{Th}$ & $\mathrm{Pa}$ & $\mathrm{U}$ & $\mathrm{Np}$ \\
\hline$S_{\mathrm{WS}}(\mathrm{Bohr})$ & 5.587 & 4.882 & 3.900 & 3.671 & 3.371 & 3.220 & 3.076 & 3.037 \\
$\gamma\left(\mathrm{J} / \mathrm{m}^{2}\right)$ & 0.07 & 0.30 & 0.82 & 1.37 & 2.33 & 2.48 & 2.65 & 2.67 \\
$W(\mathrm{eV})$ & 2.13 & 2.03 & 3.44 & 3.44 & 3.76 & 3.85 & 4.00 & 3.98 \\
\hline \hline
\end{tabular}


Communities and research project OTKA 2950 of the Hungarian National Scientific Research Foundation. The Center for Atomic-scale Materials Physics was sponsored by the Danish National Research Foundation. Part of the work was supported by grants from the Novo Nordisk Foundation and the Danish research councils through the Danish Center for Surface Reactivity.
${ }^{1}$ F. R. de Boer, R. Boom, W. C. M. Mattens, A. R. Miedema, and A. K. Niessen, Cohesion in Metals (NorthHolland, Amsterdam, 1988).

${ }^{2}$ H. B. Michaelson, J. Appl. Phys. 48, 4729 (1977).

${ }^{3}$ H. L. Skriver and N. M. Rosengaard, Phys. Rev. B 43, 9538 (1991).

${ }^{4}$ H. L. Skriver and N. M. Rosengaard, Phys. Rev. B 45, 9410 (1992).

${ }^{5}$ H. L. Skriver and N. M. Rosengaard, Phys. Rev. B 46, 7157 (1992).

${ }^{6}$ L. Vitos, J. Kollar, and H. L. Skriver Phys. Rev. B (to be published).

${ }^{7}$ M. Methfessel, D. Hennig, and M. Scheffler, Phys. Rev. B 46, 4816 (1992).

${ }^{8}$ O. K. Andersen, Phys. Rev. B 12, 3060 (1975).

${ }^{9}$ H. L. Skriver, The LMTO Method (Springer-Verlag, Berlin, 1984).

${ }^{10}$ O. Gunnarsson, O. Jepsen, and O. K. Andersen, Phys. Rev. B 27, 7144 (1983).
${ }^{11}$ O. K. Andersen and O. Jepsen, Phys. Rev. Lett. 53, 2571 (1984).

${ }^{12}$ O. K. Andersen, O. Jepsen, and D. Glötzel, in Highlights of Condensed-Matter Theory, edited by F. Bassani, F. Fumi, and M. P. Tosi (North-Holland, New York, 1985).

${ }^{13}$ O. K. Andersen, Z. Pawlowska, and O. Jepsen, Phys. Rev. B 34, 5253 (1986).

${ }^{14}$ W. R. L. Lambrecht and O. K. Andersen, Surf. Sci. 178, 256 (1986); (private communication).

${ }^{15}$ S. L. Cunningham, Phys. Rev. B 10, 4988 (1974).

16 J. Perdew and A. Zunger, Phys. Rev. B 23, 5048 (1981).

${ }^{17}$ D. M. Ceperley and B. J. Alder, Phys. Rev. Lett. 45, 566 (1980).

${ }^{18}$ W.F. Egelhoff, Surf. Sci. Rep. 6, 253 (1987).

${ }^{19}$ Y. G. Hao, O. Eriksson, G. W. Fernando, and B. R. Cooper, Phys. Rev. B 43, 9467 (1991).

${ }^{20}$ Y. G. Hao, O. Eriksson, G. W. Fernando, and B. R. Cooper, Phys. Rev. B 47, 6680 (1993). 


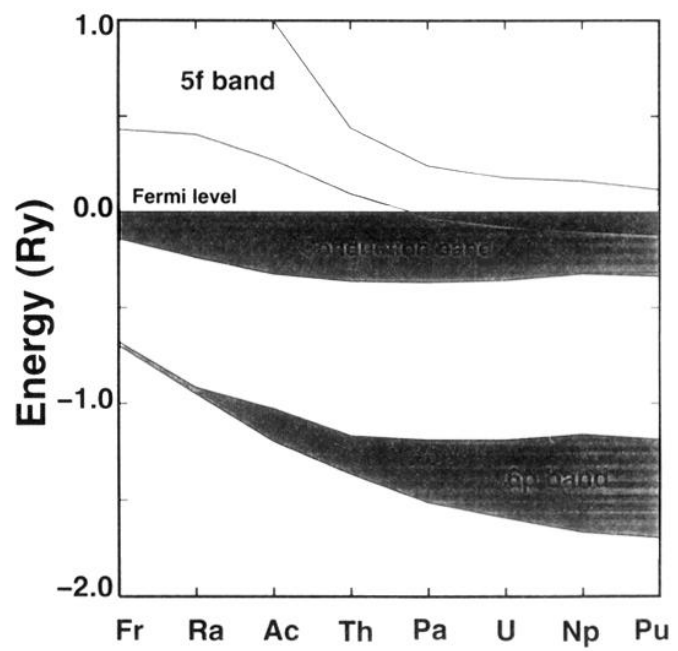

FIG. 1. The energy band structure of the light actinides. The band positions are measured from the actual Fermi level. 